\title{
26167 - A-TYPE GAMMAAMINOBUTYRIC ACID RECEPTOR (GABAAR) AND GLUTAMIC ACID DECARBOXYLASE (GAD) IN TYPE II PNEUMOCYTES
}

\section{Wei-Yang Lu PhD, Yun-Yan Xiang, PhD; William Ju; Mingyao Liu, Beverley Orser, MD PhD; Sunnybrook And Women's, Toronto, ON, Canada}

INTRODUCTION: GABAARs mediate most inhibitory neurotransmissions in the brain. Interestingly, GABAARs are also expressed in various non-neuronal tissues (1,2). Most general anesthetics increase the function of GABAARs (3). It is not known, however, whether GABAARs in non-neuronal tissues are targets for anesthetics. The mRNAs encoding GABAAR subunits are identified in type II pneumocytes (4). The main purpose of this study was to determine whether the GABAAR subunits in lung epithelial cells form functional channels.

METHODS: The expression level and subcellular location of GABAAR subunits and GAD in A549 cell, a widely-used type II human lung epithelial cell line, were investigated using Western blots and immunocytochemistry. Patch clamp electrophysiology was also used to study receptor function.

RESULTS: Proteins of GABAAR á; 2 and ä; subunits and GAD-65 were determined in A549 cells. Clusters of GABAAR subunits were localized along with the plasma membrane, whereas GAD-65, a GABA metabolism regulating enzyme, was identified to be associated with vesicular structures. Application of GABA evoked fast transmembrane currents, which were effectively inhibited by the GABAAR antagonist picrotoxin, and significantly enhanced by propofol (2 ìm).

DISCUSSION: Type II pneumocytes express functional GABAARs that are subject to propofol modulation. Also the cell may release GABA via vesicle-mediated exocytosis, regulating the cell function as an autocrine / paracrine modulator.

\section{REFERENCES:}

1.Diabetes. 1991 Nov; 40 (11):1365-74

2.Am J Physiol Gastrointest Liver Physiol. 2001 Aug; 281 (2):G311-5.

3.Trends Pharmacol Sci. 2005 Oct;26 (10):503-10

4.Biochem Biophys Res Commun. 2004 Jul 2;319 (3):774-80 